University of Wollongong

Research Online

Faculty of Engineering and Information

Faculty of Engineering and Information

Sciences - Papers: Part B

Sciences

2018

Sharpness results concerning finite differences in Fourier analysis on the circle group

Rodney Nillsen

University of Wollongong, nillsen@uow.edu.au

Susumu Okada

Follow this and additional works at: https://ro.uow.edu.au/eispapers1

Part of the Engineering Commons, and the Science and Technology Studies Commons

Research Online is the open access institutional repository for the University of Wollongong. For further information contact the UOW Library: research-pubs@uow.edu.au 


\title{
Sharpness results concerning finite differences in Fourier analysis on the circle group
}

\author{
Abstract \\ Let $\mathrm{G}$ denote the group or , let denote the identity element of $\mathrm{G}$, and let $\mathrm{s}$ be given. Then, a \\ lemph\{difference of order\} $s$ is a function $f L 2(G)$ for which there are $a G$ and $g L 2(G)$ such that $f=(-a) s g$. \\ Let $s(L 2(G))$ be the vector space of functions that are finite sums of differences of order $s$. It is known \\ that if $f L 2(), f s(L 2())$ if and only if $-f(x) 2 x-2 s d x$. Also, if $f L 2(), f s(L 2())$ if and only if $f(0)=0$. Consequently, \\ $s(L 2(G))$ is a Hilbert space in a (possibly) weighted L2-norm. It is known that every function in $s(L 2(G))$ is \\ a sum of $2 s+1$ differences of order $s$. However, there are functions in $s(L 2())$ that are not a sum of $2 s$ \\ differences of order $s$, and we call the latter type of fact a lemph\{sharpness result\}. In 1(L2()), it is known \\ that there are functions that are not a sum of two differences of order one. A main aim here is to obtain \\ new sharpness results in the spaces $s(L 2())$ that complement the results known for, but also to present \\ new results in $s(L 2())$ that do not correspond to known results in s(L2()). Some results are obtained using \\ connections with Diophantine approximation. The techniques also use combinatorial estimates for \\ potentials arising from points in the unit cube in Euclidean space, and make use of subtraction sets in \\ arithmetic combinatorics.

\section{Disciplines} \\ Engineering | Science and Technology Studies

\section{Publication Details} \\ Nillsen, R. \& Okada, S. (2018). Sharpness results concerning finite differences in Fourier analysis on the \\ circle group. Acta Universitatis Szegediensis: Acta Scientiarum Mathematicarum, 84 (3-4), 591-609.
}




\title{
Sharpness results concerning finite differences in Fourier analysis on the circle group
}

\author{
A preprint of a paper in Acta Sci. Math. (Szeged), 84 (2018), 591-609
}

\author{
RODNEY NILLSEN and SUSUMU OKADA
}

\begin{abstract}
Let $G$ denote the group $\mathbb{R}$ or $\mathbb{T}$, let $e$ denote the identity element of $G$, and let $s \in \mathbb{N}$ be given. Then, a difference of order $s$ is a function $f \in L^{2}(G)$ for which there are $b \in G$ and $g \in L^{2}(G)$ such that $f=\left(\delta_{e}-\delta_{b}\right)^{s} * g$. Let $\mathcal{D}_{s}\left(L^{2}(G)\right)$ be the vector space of functions that are finite sums of differences of order $s$. It is known that if $f \in L^{2}(\mathbb{R})$, $f \in \mathcal{D}_{s}\left(L^{2}(\mathbb{R})\right)$ if and only if $\int_{-\infty}^{\infty}|\widehat{f}(x)|^{2}|x|^{-2 s} d x<\infty$. Also, if $f \in L^{2}(\mathbb{T}), f \in \mathcal{D}_{s}\left(L^{2}(\mathbb{T})\right)$ if and only if $\widehat{f}(0)=0$. Consequently, $\mathcal{D}_{s}\left(L^{2}(G)\right)$ is a Hilbert space in a (possibly) weighted $L^{2}$-norm. It is known that every function in $\mathcal{D}_{s}\left(L^{2}(G)\right)$ is a sum of $2 s+1$ differences of order $s$. However, there are functions in $\mathcal{D}_{s}\left(L^{2}(\mathbb{R})\right)$ that are not a sum of $2 s$ differences of order $s$, and we call the latter type of fact a sharpness result. In $\mathcal{D}_{1}\left(L^{2}(\mathbb{T})\right)$, it is known that there are functions that are not a sum of two differences of order one. A main aim here is to obtain new sharpness results in spaces $\mathcal{D}_{s}\left(L^{2}(\mathbb{T})\right)$ that complement the results known for $\mathbb{R}$, but also to present new results in $\mathcal{D}_{s}\left(L^{2}(\mathbb{T})\right)$ that do not correspond to known results in $\mathcal{D}_{s}\left(L^{2}(\mathbb{R})\right)$. Some results are obtained using connections with Diophantine approximation. The techniques also use combinatorial estimates for potentials arising from points in the unit cube in Euclidean space, and make use of subtraction sets in arithmetic combinatorics.
\end{abstract}

\section{Introduction}

Let $\mathbb{N}, \mathbb{R}, \mathbb{C}$ and $\mathbb{Z}$ respectively denote the sets of natural, real and complex numbers and the integers. Put $\mathbb{T}=\{z: z \in \mathbb{C}$ and $|z|=1\}$. Then, $\mathbb{T}$ can be identified with $[0,2 \pi)$ by means of the mapping $t \longmapsto e^{i t}$ from $[0,2 \pi)$ onto $\mathbb{T}$. The normalised Lebesgue measure on $\mathbb{T}$ is denoted by $\mu$. If $G$ is either $\mathbb{R}$ or $\mathbb{T}$, let $L^{2}(G)$ be the usual space of square integrable functions, let $M(G)$ denote the bounded regular Borel measures on $G$, let $\delta_{x}$ be the Dirac measure at any point $x \in G$, and let $*$ denote convolution. If $\lambda \in M(G), \lambda^{n}$ denotes $\lambda * \lambda * \cdots * \lambda$, where $\lambda$ appears $n$ times. If $s \in \mathbb{N}$ and $e$ denotes the identity in $G$, we define a difference of order $s$ to be a function in $L^{2}(G)$ of the form $\left(\delta_{e}-\delta_{a}\right)^{s} * f$ for some $a \in G$ and $f \in L^{2}(G)$. A difference of order 1 has the form $g-\delta_{b} * g$ for some $b \in G$ and $g \in L^{2}(G)$, and a difference of order 2 has the form $h-2^{-1}\left(\delta_{c}+\delta_{-c}\right) * h$ for some $c \in G$ and $h \in L^{2}(G)$. Note that if

AMS subject classifications: 42A16, 52AA40

Key words and phrases: Fourier transform, finite differences, subspaces of $L^{2}(\mathbb{T})$, combinatorial inequalities, badly approximable vectors in $\mathbb{R}^{n}$, sharpness results, Sobolev spaces

The first named author received support for some of this research from the Institute of Mathematics and its Applications at the University of Wollongong. 
$t<s$, a difference of order $s$ is a difference of order $t$. The vector subspace of $L^{2}(G)$ finitely spanned by the differences of order $s$ is denoted by $\mathcal{D}_{s}\left(L^{2}(G)\right)$. The Fourier transform of a function $f \in L^{2}(G)$ is denoted by $\widehat{f}$. Thus, for $f \in L^{2}(\mathbb{R}), \widehat{f}$ is given by the extension to all of $L^{2}(\mathbb{R})$ of the transform

$$
\widehat{g}(x)=\int_{-\infty}^{\infty} g(t) e^{-i x t} d t, \text { for } x \in \mathbb{R}
$$

which is defined for all $g \in L^{1}(\mathbb{R}) \cap L^{2}(\mathbb{R})$. If $f \in L^{2}(\mathbb{T}), \widehat{f}$ is given by

$$
\widehat{f}(n)=\frac{1}{2 \pi} \int_{0}^{2 \pi} f(t) e^{-i n t} d t, \text { for } n \in \mathbb{Z} .
$$

In [8, Theorem 2], Meisters and Schmidt showed that if $T$ is in the algebraic dual of $L^{2}(\mathbb{T})$ and $T\left(\delta_{b} * g\right)=T(g)$ for all $b \in \mathbb{T}$ and $g \in L^{2}(\mathbb{T})$, then $T$ is continuous on $L^{2}(\mathbb{T})$. This is an immediate consequence of the fact that $\mathcal{D}_{1}\left(L^{2}(\mathbb{T})\right)$ has codimension 1 in $L^{2}(\mathbb{T})$ and, in fact, they proved in $[8$, Theorem 1$]$ that

$$
\mathcal{D}_{1}\left(L^{2}(\mathbb{T})\right)=\left\{f: f \in L^{2}(\mathbb{T}) \text { and } \int_{\mathbb{T}} f d \mu=0\right\}
$$

It was also proved in [8, Theorems 1 and 5] that every function in $\mathcal{D}_{1}\left(L^{2}(\mathbb{T})\right)$ is a sum of three first order differences, but that there are functions in $\mathcal{D}_{1}\left(L^{2}(\mathbb{T})\right)$ that are not a sum of two first order differences. Thus, three is sharp for the minimum number of needed differences in $\mathcal{D}_{1}\left(L^{2}(\mathbb{T})\right)$. Subsequently it was proved in $\left[9\right.$, Theorem 8] that $\mathcal{D}_{s}\left(L^{2}(\mathbb{T})\right)=\mathcal{D}_{1}\left(L^{2}(\mathbb{T})\right)$ for all $s \in \mathbb{N}$, and that any function in $\mathcal{D}_{s}\left(L^{2}(\mathbb{T})\right)$ is a sum of $2 s+1$ differences of order $s$. However, the question of the sharpness of $2 s+1$ for the minimum number of differences needed in $\mathcal{D}_{s}\left(L^{2}(\mathbb{T})\right)$ was unresolved.

When it comes to the group $\mathbb{R}$ rather than $\mathbb{T}$, it was proved in $[9$, Theorem 2$]$, and see also [10, Proposition 5.2], that for $s \in \mathbb{N}$,

$$
\mathcal{D}_{s}\left(L^{2}(\mathbb{R})\right)=\left\{f: f \in L^{2}(\mathbb{R}) \text { and } \int_{-\infty}^{\infty} \frac{|\widehat{f}(x)|^{2}}{|x|^{2 s}} d x<\infty\right\},
$$

and that every function in $\mathcal{D}_{s}\left(L^{2}(\mathbb{R})\right)$ is a sum of $2 s+1$ differences of order $s$. A more approachable exposition of this result is in [11]. In [12, Theorem 4.8], it was shown that $2 s+1$ is sharp, in that there are functions in $\mathcal{D}_{s}\left(L^{2}(\mathbb{R})\right)$ that are not a sum of $2 s$ differences of order $s$.

A purpose of this paper is to resolve the sharpness question for the spaces $\mathcal{D}_{s}\left(L^{2}(\mathbb{T})\right.$ ), by proving that there are functions in $\mathcal{D}_{s}\left(L^{2}(\mathbb{T})\right)$ that are not a sum of $2 s$ differences of order $s$. As well, a purpose is to give some specific examples of such functions. Also, related results are obtained. Note that the function 0 is a difference of any order. Consequently, if $t \in \mathbb{N}$ and $f$ is a sum of $t$ differences of order $s$ and $u \in \mathbb{N}$ with $u>t$, then $f$ is also a sum of $u$ differences of order $s$. The techniques call upon those used in [12], but provide additional insight and generality. Also, further results not pursued for $L^{2}(\mathbb{R})$ in [12] are obtained for $L^{2}(\mathbb{T})$. The functions are constructed by defining their Fourier transforms on subsets of $\mathbb{Z}$ 
of the form $(A-A)_{+}$, where $A \subseteq \mathbb{N}$ and $(A-A)_{+}=\{x-y: x, y \in A$ and $x-y>0\}$. Sets of the form $(A-A)_{+}$are known as "partial difference sets" in arithmetic combinatorics. In fact, we take the set $A \subseteq \mathbb{N}$ to be of the form $\cup_{n=1}^{\infty} A_{n}$ where each $A_{n}$ is finite and $A_{n} \subseteq A_{n+1}$ for all $n$, and the construction of examples depends upon the rate of growth of the sizes of the sets $\left(A_{n}-A_{n}\right)_{+}$. A case of particular interest arises from the fact that if $F$ is a finite subset of $\mathbb{N}$ with a specified number of elements, then the number of elements of $(F-F)_{+}$ is a minimum if and only if the elements of $F$ form an arithmetic progression.

\section{Definitions and preparation}

If $x \in \mathbb{R}, d_{\mathbb{Z}}(x)$ denotes the distance from $x$ to the nearest integer. Thus,

$$
d_{\mathbb{Z}}(x)=\min \{|r-x|: r \in \mathbb{Z}\} .
$$

Lemma 2.1. If $x \in \mathbb{R}$,

$$
2 d_{\mathbb{Z}}(x) \leq|\sin \pi x| \leq \pi d_{\mathbb{Z}}(x) .
$$

Proof. This follows from the definition of $d_{\mathbb{Z}}(x)$ and properties of the sine function. See [10, page 89] or [17, pages 165-166].

If $u \in \mathbb{R}$, put $e(u)=e^{i u}$. Then, if $a \in \mathbb{R}, \mathbb{T}=\{e(u): u \in[a, a+2 \pi)\}$. Let $M(\mathbb{T})$ be the set of bounded regular Borel measures on $\mathbb{T}$, which may be identified with $M([0,2 \pi))$, the set of bounded regular Borel measures on $[0,2 \pi)$, by means of the mapping $A \longmapsto \lambda\left(e^{i A}\right)$ for Borel sets $A$ in $[0,2 \pi)$. The Fourier transform of $\lambda \in M(\mathbb{T})$ is given by

$$
\widehat{\lambda}(n)=\int_{\mathbb{T}} e(-u n) d \lambda(u), \text { for } n \in \mathbb{Z} .
$$

If $\lambda=f \in L^{2}(\mathbb{T})$, then $\widehat{f} \in L^{2}(\mathbb{T})$ and

$$
\widehat{f}(n)=\frac{1}{2 \pi} \int_{0}^{2 \pi} e^{-i n t} f\left(e^{i t}\right) d t, \text { for } n \in \mathbb{Z} .
$$

In what follows we will consider series of non-negative terms, some of which may be of the form $a / 0$, for some $a>0$. We then take $a / 0$ to be $\infty$ so that any such series will be divergent. Any expression of the form $0 / 0$ is taken to be 0 .

Theorem 2.1. Let $f \in L^{2}(\mathbb{T})$ and let $\mu_{1}, \mu_{2}, \ldots, \mu_{t} \in M(\mathbb{T})$. Then the following conditions (i) and (ii) are equivalent.

(i) There are $f_{1}, f_{2}, \ldots, f_{t} \in L^{2}(\mathbb{T})$ such that $f=\sum_{j=1}^{t} \mu_{j} * f_{j}$.

(ii)

$$
\sum_{n=-\infty}^{\infty} \frac{|\widehat{f}(n)|^{2}}{\sum_{j=1}^{t}\left|\widehat{\mu_{j}}(n)\right|^{2}}<\infty .
$$

Also, the following holds. 
(iii) If $s \in \mathbb{N}$ is given and $f \in L^{2}(\mathbb{T})$, then $f$ is a sum of $t$ differences of order $s$ if and only if there are $a_{1}, a_{2}, \ldots, a_{t} \in \mathbb{R}$ such that

$$
\sum_{n=-\infty}^{\infty} \frac{|\widehat{f}(n)|^{2}}{\sum_{j=1}^{t} d_{\mathbb{Z}}\left(a_{j} n\right)^{2 s}}<\infty
$$

In this case, there are $g_{1}, g_{2}, \ldots, g_{t} \in L^{2}(\mathbb{T})$ such that

$$
f=\sum_{j=1}^{t}\left(\delta_{1}-\delta_{e\left(-2 \pi a_{j}\right)}\right)^{s} * g_{j} .
$$

Proof. For the equivalence of (i) and (ii) see [8] or [10, page 23].

In order to prove (2.1), note that $f$ is a sum of $t$ differences of order $s$ if and only if there are $a_{1}, a_{2}, \ldots, a_{t} \in \mathbb{R}$ and $g_{1}, g_{2}, \ldots, g_{t}$ such that

$$
f=\sum_{j=1}^{t}\left(\delta_{1}-\delta_{e\left(-2 \pi a_{j}\right)}\right)^{s} * g_{j} .
$$

Now, observe that for $a \in \mathbb{R}, \widehat{\delta}_{e(a)}(n)=e^{-i n a}$. Thus, we see that

$$
\left(\left(\delta_{1}-\delta_{e\left(-2 \pi a_{j}\right)}\right)^{s}\right)^{\wedge}(n)=\left(\left(\delta_{1}-\delta_{e\left(-2 \pi a_{j}\right)}\right)^{\wedge}(n)\right)^{s}=\left(1-e^{2 \pi i n a_{j}}\right)^{s} .
$$

Consequently, if $f \in L^{2}(\mathbb{T})$, the equivalence of (i) and (ii), together with (2.3) and (2.4), give that $f$ is a sum of $t$ differences of order $s$ if and only if there are $a_{1}, a_{2}, \ldots, a_{t} \in \mathbb{R}$ such that

$$
\sum_{n=-\infty}^{\infty} \frac{|\widehat{f}(n)|^{2}}{\sum_{j=1}^{t}\left|1-e^{2 i \pi a_{j} n}\right|^{2 s}}<\infty
$$

As $\left|1-e^{2 \pi i a}\right|=2|\sin \pi a|$, we see that $f$ is a sum of $t$ differences of order $s$ if and only if

$$
\sum_{n=-\infty}^{\infty} \frac{|\widehat{f}(n)|^{2}}{\sum_{j=1}^{t}\left|\sin \pi a_{j} n\right|^{2 s}}<\infty,
$$

for some $a_{1}, a_{2}, \ldots, a_{t} \in \mathbb{R}$. The inequality (2.1) now follows from Lemma 2.1.

Conversely, if the series in (2.1) is convergent, it follows from Lemma 2.1, along the lines above, that the series in (2.5) is convergent. Then, reasoning backwards from before, using (2.3), (2.4) and the equivalence of (i) and (ii), we obtain (2.2).

\section{Sobolev spaces and differences in $L^{2}(\mathbb{T})$}

The Sobolev space $W_{s}\left(L^{2}(\mathbb{T})\right)$, for $s \in \mathbb{N}$, consists of those $f \in L^{2}(\mathbb{T})$ such that $\sum_{n \in \mathbb{Z}}|n|^{2 s}|\widehat{f}(n)|^{2}<$ $\infty$. Let $D$ denote the differentiation operator and let $D^{s}$ denote the composition of $D$ with itself, taken $s$ times. Then, in the sense of distributions, $D^{s}$ maps $W_{s}\left(L^{2}(\mathbb{T})\right)$ into $L^{2}(\mathbb{T})$ 
and its range is $\mathcal{D}_{s}\left(L^{2}(\mathbb{T})\right)$. Now, considering the sums in (2.1), one approach might be to estimate the numerator and denominator separately, with the aim of deriving conditions either on $f$ or $a_{1}, a_{2}, \ldots, a_{t}$. However, in general, the convergence of a series such as in (2.1) involves a more subtle interaction. In this section we simply note that appropriate assumptions about the denominator in (2.1) lead immediately to results along the lines of: if $f \in L^{2}(\mathbb{T})$ is in a space $W_{s}\left(L^{2}(\mathbb{T})\right)$ and $\widehat{f}(0)=0$, then $f$ may be expressed as a sum of a certain number of differences of order $s$. These "appropriate assumptions" derive from results in Diophantine approximation.

Definition. Let $r \in \mathbb{N}$ and let $\theta \in[0, \infty)$. Then we define $B(r, \theta)$ to be the set of all $b=\left(b_{1}, b_{2}, \ldots, b_{r}\right) \in \mathbb{R}^{r}$ such that for some $c(b, \theta)>0$,

$$
\max \left\{\left|b_{j}-\frac{p_{j}}{q}\right|: 1 \leq j \leq r\right\}>\frac{c(b, \theta)}{|q|^{\theta+1+1 / r}},
$$

for all $p_{1}, p_{2}, \ldots, p_{r} \in \mathbb{Z}$ and all $q \in \mathbb{Z}$ with $q \neq 0$.

In considering the above definition, note the fact that if $b \in \mathbb{R}^{r}$, there are infinitely many $p_{1}, p_{2}, \ldots, p_{r}, q \in \mathbb{Z}$ with $q \neq 0$ such that $\left|b_{j}-p_{j} / q\right|<1 /|q|^{1+1 / r}$, for all $j=1,2, \ldots, r$ (see [13, page 47] for example). Membership of $b$ in $B(r, \theta)$ assigns a type of "measure of approximability" of $b$ by rational numbers. If $b \in B(r, \theta)$ then $b_{j}$ is irrational for at least one $j \in\{1,2, \ldots, r\}$. As well, if $\theta, \theta^{\prime} \in[0, \infty)$ with $\theta<\theta^{\prime}$, then $B(r, \theta) \subseteq B\left(r, \theta^{\prime}\right)$. Following [16, page 42], we say that a vector $b \in \mathbb{R}^{r}$ is badly approximable if it is in $B(r, 0)$. A vector $b \in \mathbb{R}^{r}$ is called a Liouville vector if for all $n \in \mathbb{N}$, there are $p_{1}, p_{2}, \ldots, p_{r} \in \mathbb{Z}$ and $q \in \mathbb{Z}$ with $q \neq 0$ such that $0<\max \left\{\left|b_{j}-p_{j} / q\right|: 1 \leq j \leq r\right\}<1 /|q|^{n}$. In the case $r=1$, the Liouville vectors are the Liouville numbers, as defined in [14, page 7]. If $b \in \mathbb{R}^{r}$ is a Liouville vector, at least one of the numbers $b_{1}, b_{2}, \ldots, b_{r}$ is Liouville. So, as the Liouville numbers have measure zero [14, page 7], the Liouville vectors also have measure zero in $\mathbb{R}^{r}$. The space $\mathbb{R}^{r}$ is the disjoint union of the vectors that have only rational cordinates, the Liouville vectors, and the set $\cup_{\theta \in[0, \infty)} B(r, \theta)$, and this latter set has full measure in $\mathbb{R}^{r}$.

Now if $f \in L^{2}(\mathbb{T})$ with $\widehat{f}(0)=0$, note that $f$ is a sum of $2 s+1$ differences of order $s$, by Theorem 8 in [9].

Theorem 3.1. Let $r, s \in \mathbb{N}$ and $\theta \in[0, \infty)$. Let $b=\left(b_{1}, \ldots, b_{r}\right) \in B(r, \theta / 2 s)$. Let $f \in L^{2}(\mathbb{T})$ be such that

$$
\sum_{n \in \mathbb{Z}}|\widehat{f}(n)|^{2}|n|^{2 s / r+\theta}<\infty
$$

Then, if $\widehat{f}(0)=0$, there are functions $f_{1}, \ldots, f_{r} \in L^{2}(\mathbb{T})$ such that $f=\sum_{j=1}^{r}\left(\delta_{1}-\delta_{e\left(-2 \pi b_{j}\right)}\right)^{s} * f_{j}$, and consequently $f$ is a sum of $r$ differences of order $s$.

Proof. As $b \in B(r, \theta / 2 s)$, there is $c(b, \theta / 2 s)>0$ such that (3.1) holds with $\theta / 2 s$ replacing $\theta$. Now, let $q \in \mathbb{Z}$ with $q \neq 0$, and let $p_{1}, \ldots, p_{r} \in \mathbb{Z}$ be such that $d_{\mathbb{Z}}\left(b_{j} q\right)=\left|b_{j} q-p_{j}\right|$, for $j=1,2, \ldots, r$. Then,

$$
\frac{1}{|q|} \sum_{j=1}^{r} d_{\mathbb{Z}}\left(b_{j} q\right)=\sum_{j=1}^{r}\left|b_{j}-\frac{p_{j}}{q}\right| \geq \max \left\{\left|b_{j}-\frac{p_{j}}{q}\right|: 1 \leq j \leq r\right\} \geq \frac{c(b, \theta / 2 s)}{|q|^{\theta / 2 s+1+1 / r}} .
$$


So, there is $d(b, \theta, s)>0$ such that for all $q \in \mathbb{Z}$ with $q \neq 0$,

$$
\sum_{j=1}^{r} d_{\mathbb{Z}}\left(b_{j} q\right)^{2 s} \geq \frac{d(b, \theta, s)}{|q|^{2 s / r+\theta}}
$$

We now have

$$
\sum_{q \in \mathbb{Z}, q \neq 0} \frac{|\widehat{f}(q)|^{2}}{\sum_{j=1}^{r} d_{\mathbb{Z}}\left(b_{j} q\right)^{2 s}} \leq \frac{1}{d(b, \theta, s)} \sum_{\substack{q \in \mathbb{Z} \\ q \neq 0}}|\widehat{f}(q)|^{2}|q|^{2 s / r+\theta}<\infty .
$$

So, by Theorem 2.1 , there are $f_{1}, \ldots, f_{r} \in L^{2}(\mathbb{T})$ such that $f=\sum_{j=1}^{r}\left(\delta_{1}-\delta_{e\left(-2 \pi b_{j}\right)}\right)^{s} * f_{j}$.

Corollary 3.1. Let $r, s \in \mathbb{N}$, let $b \in \mathbb{R}^{r}$ be badly approximable, and let $f \in L^{2}(\mathbb{T})$ be such that $\widehat{f}(0)=0$ and $\sum_{n \in \mathbb{Z}}|\widehat{f}(n)|^{2}|n|^{2 s / r}<\infty$. Then, there are $f_{1}, f_{2}, \ldots, f_{r} \in L^{2}(\mathbb{T})$ such that $f=\sum_{j=1}^{r}\left(\delta_{1}-\delta_{e\left(-2 \pi b_{j}\right)}\right)^{s} * f_{j}$, and $f$ is a sum of $r$ differences of order $s$.

Corollary 3.2. Let $f \in L^{2}(\mathbb{T}), s \in \mathbb{N}, \widehat{f}(0)=0$ and $\sum_{n \in \mathbb{Z}}|\widehat{f}(n)|^{2}|n|^{2 s}<\infty$. Then, $f$ is a difference of order $s$.

The observations above raise the question of the properties of the sets $B(r, \theta)$. It is well known that the badly approximable elements of $\mathbb{R}^{r}$ are a set of measure zero, a result that is a consequence of a result of Khintchine, discussed and extended in [16, pages 60-61]. Earlier, Cassels [5] had shown that the badly approximable vectors in $\mathbb{R}^{r}$ form a continuum, and a proof based upon "the $(\alpha, \beta)$ game" may be found also in [16, page 54]. It was shown also in $\left[4\right.$, Theorem III, page 79] that if the real numbers $1, b_{1}, b_{2}, \ldots, b_{r}$ are linearly independent elements of an algebraic real number field of dimension $r+1$ over $\mathbb{Q}$, then $b=\left(b_{1}, b_{2}, \ldots, b_{r}\right)$ is badly approximable in $\mathbb{R}^{r}$. Badly approximable vectors in $\mathbb{R}^{r}$ are also being used in deconvolution problems in signal precessing, see [6], [15], where the latter has a succinct discussion of results on badly approximable vectors. When $r=1$, a good deal is known about sets related to the sets $B(1, \theta)$. Further information is in [1, pages 171-172], [2] and [3, pages 246-248], where there are more references.

\section{Sharp results for $L^{2}(\mathbb{T})$}

Lemma 4.1. Let $p, q \in \mathbb{N}$ with $p<q$. Let $a_{q} \geq 0$ and $b_{p}+b_{p+1}+\ldots+b_{q} \geq 0$. Then,

$$
\sum_{j=p}^{q} a_{j} b_{j} \geq \sum_{j=p}^{q-1}\left(a_{j}-a_{j+1}\right)\left(b_{p}+\cdots+b_{j}\right) .
$$

Proof. It can be proved by induction or rearrangement of terms that

$$
\sum_{j=p}^{q} a_{j} b_{j}=\left[\sum_{j=p}^{q-1}\left(a_{j}-a_{j+1}\right)\left(b_{p}+\cdots+b_{j}\right)\right]+a_{q}\left(\sum_{j=p}^{q} b_{j}\right) .
$$

The inequality is immediate. Also, see [12, page 492]. 
Definitions. If $A$ is a finite set, $|A|$ denotes the number of elements in $A$. Let $A, B \subseteq \mathbb{N}$ be non-empty. Then, we define

$$
A-B=\{a-b: a \in A, b \in B\} \text { and }(A-B)_{+}=\{x: x \in A-B \text { and } x>0\} .
$$

Note that if $A$ has a single element, $(A-A)_{+}=\emptyset$.

A set $(A-B)_{+}$as defined above is a special type of partial sum set, as discussed in [17, section 2.5]. The following observation corresponds to part of Lemma 2.1 in [17, page 54].

Lemma 4.2. Let $F$ be a finite subset of $\mathbb{N}$ having $n$ elements. Then, $(F-F)_{+}$has at least $n-1$ elements and at most $n(n-1) / 2$ elements, and every element of $(F-F)_{+}$can be written in the form $a-b$ for some $a, b \in F$ in at most $n-1$ ways.

Proof If we subtract each of the smaller elements from the largest element we obtain $n-1$ elements of $(F-F)_{+}$. Also, there are at most $n(n-1) / 2$ pairs $(a, b)$ with $a, b \in F$ such that $a<b$, so there are at most $n(n-1) / 2$ elements in $(F-F)_{+}$. Now, for a given $b \in F$ that is not the least element of $F$, the numbers $b-a$ are distinct for all values of $a \in F$ with $a<b$. As there are at most $n-1$ possible values of $b$, we see that there are at most $n-1$ pairs $(a, b)$ such that $a-b$ is equal to a given element of $(F-F)_{+}$.

Lemma 4.3. Let $r, r^{\prime} \in \mathbb{N}$. Then the following hold.

(i) If $r^{\prime}<r$, there is $G\left(r, r^{\prime}\right)>0$ such that for any $n \in \mathbb{N}$ with $n \geq 2$ and $x_{1}, x_{2}, \ldots, x_{n} \in$ $[0,1]^{r^{\prime}}$

$$
\sum_{1 \leq i<j \leq n} \frac{1}{\left|x_{i}-x_{j}\right|^{r}} \geq G\left(r, r^{\prime}\right) n^{1+r / r^{\prime}} .
$$

(ii) There is $H(r)>0$ such that for any $n \in \mathbb{N}$ with $n \geq 2$ and $x_{1}, x_{2}, \ldots, x_{n}$ in $[0,1]^{r}$,

$$
\sum_{1 \leq i<j \leq n} \frac{1}{\left|x_{i}-x_{j}\right|^{r}} \geq H(r) n^{2} \log n .
$$

Proof. The above are the statements (ii) and (iii) in Theorem 3.4 of [12].

Lemma 4.4. The following hold.

(i) Let $r, r^{\prime} \in \mathbb{N}$ with $r^{\prime}<r$. Then there is a constant $J\left(r, r^{\prime}\right)>0$ with the following property: if $n \in \mathbb{N}$ with $n \geq 2$ and if $C$ is a finite subset of $\mathbb{N}$ with $|C|=n$, then for all $b_{1}, \ldots, b_{r^{\prime}} \in \mathbb{R}$

$$
\sum_{j \in(C-C)_{+}} \frac{1}{\sum_{i=1}^{r^{\prime}} d_{\mathbb{Z}}\left(j b_{i}\right)^{r}} \geq J\left(r, r^{\prime}\right) n^{r / r^{\prime}} .
$$

(ii) Let $r \in \mathbb{N}$. Then there is a constant $K(r)>0$ with the following property: if $n \in \mathbb{N}$ with $n \geq 2$ and if $C$ is a finite subset of $\mathbb{N}$ with $|C|=n$, then for all $b_{1}, \ldots, b_{r^{\prime}} \in \mathbb{R}$,

$$
\sum_{j \in(C-C)_{+}} \frac{1}{\sum_{i=1}^{r} d_{\mathbb{Z}}\left(j b_{i}\right)^{r}} \geq K(r) n \log n .
$$


Proof. (i) Let $C \subseteq \mathbb{N}$, let $|C|=n \geq 2$ and let $b_{1}, b_{2}, \ldots, b_{r^{\prime}} \in \mathbb{R}$. Using Lemma 4.2, we see that every element of $(C-C)_{+}$can be expressed in at most $n-1$ ways as $k-\ell$ with $k, \ell \in C$, and so we have

$$
\begin{aligned}
\sum_{k, \ell \in C, \ell<k}\left(\sum_{i=1}^{r^{\prime}} d_{\mathbb{Z}}\left((k-\ell) b_{i}\right)^{r}\right)^{-1} & =\sum_{j \in(C-C)_{+}} \sum_{k, \ell \in C, k-\ell=j}\left(\sum_{i=1}^{r^{\prime}} d_{\mathbb{Z}}\left(j b_{i}\right)^{r}\right)^{-1} \\
& \leq n \sum_{j \in(C-C)_{+}}\left(\sum_{i=1}^{r^{\prime}} d_{\mathbb{Z}}\left(j b_{i}\right)^{r}\right)^{-1} .
\end{aligned}
$$

Now, for $u \in \mathbb{R}$, let $\operatorname{fr}(u)$ denote its fractional part. Put, for $k \in \mathbb{N}$,

$$
x_{k}=\left(\operatorname{fr}\left(k b_{1}\right), \ldots, \operatorname{fr}\left(k b_{r^{\prime}}\right)\right) \in[0,1]^{r^{\prime}} .
$$

Let $G\left(r, r^{\prime}\right)>0$ be as in (i) of Lemma 4.3. Then, as $|C|=n$,

$$
\sum_{i, j \in C, i<j} \frac{1}{\left|x_{i}-x_{j}\right|^{r}} \geq G\left(r, r^{\prime}\right) n^{1+r / r^{\prime}}
$$

Observe that $|\operatorname{fr}(u)-\operatorname{fr}(v)| \geq d_{\mathbb{Z}}(u-v)$ for all $u, v \in \mathbb{R}$. Thus,

$$
\left|x_{k}-x_{\ell}\right|=\left(\sum_{i=1}^{r^{\prime}}\left(\operatorname{fr}\left(k b_{i}\right)-\operatorname{fr}\left(\ell b_{i}\right)\right)^{2}\right)^{1 / 2} \geq\left(\sum_{i=1}^{r^{\prime}} d_{\mathbb{Z}}\left((k-\ell) b_{i}\right)^{2}\right)^{1 / 2}
$$

Also, a compactness argument shows that there is $E\left(r, r^{\prime}\right)>0$ such that for all $u_{1}, u_{2}, \ldots, u_{r^{\prime}} \in$ $\mathbb{R}$

$$
\left(\sum_{i=1}^{r^{\prime}}\left|u_{i}\right|^{2}\right)^{r / 2} \geq E\left(r, r^{\prime}\right) \sum_{i=1}^{r^{\prime}}\left|u_{i}\right|^{r}
$$

Using these facts, together with (4.2) and (4.3), we have

$$
\begin{aligned}
\sum_{j \in(C-C)_{+}}\left(\sum_{i=1}^{r^{\prime}}\left(d_{\mathbb{Z}}\left(j b_{i}\right)^{r}\right)^{-1}\right. & \geq n^{-1} \sum_{k, \ell \in C, \ell<k}\left(\sum_{i=1}^{r^{\prime}}\left(d_{\mathbb{Z}}(k-\ell) b_{i}\right)^{r}\right)^{-1} \\
& \geq n^{-1} E\left(r, r^{\prime}\right) \sum_{k, \ell \in C, \ell<k} \frac{1}{\left|x_{k}-x_{\ell}\right|^{r}} \\
& \geq E\left(r, r^{\prime}\right) G\left(r, r^{\prime}\right) n^{-1} n^{1+r / r^{\prime}} \\
& =J\left(r, r^{\prime}\right) n^{r / r^{\prime}}
\end{aligned}
$$

where $J\left(r, r^{\prime}\right)=E\left(r, r^{\prime}\right) G\left(r, r^{\prime}\right)$.

To prove (ii), put $r=r^{\prime}$ in the above argument and proceed on the same lines, except that at (4.3) we use (ii) instead of (i) in Lemma 4.3. 
We now proceed to use Lemma 4.4 to construct certain functions in $L^{2}(\mathbb{T})$ that cannot be expressed as a sum of a certain number of differences. Let $\left(A_{n}\right)_{n=1}^{\infty}$ be an increasing sequence of non-empty finite subsets of $\mathbb{Z}$ and put $A=\cup_{n=1}^{\infty} A_{n}$. Put

$$
D_{1}=\left(A_{1}-A_{1}\right)_{+} \text {and } D_{n}=\left(A_{n}-A_{n}\right)_{+} \cap\left(A_{n-1}-A_{n-1}\right)_{+}^{c} \text {, for } n \geq 2 .
$$

Note that if $A_{1}$ consists of a single point, $D_{1}=\emptyset$. Since $\left(A_{n}\right)_{n=1}^{\infty}$ is increasing we have

$$
(A-A)_{+}=\bigcup_{n=1}^{\infty}\left(A_{n}-A_{n}\right)_{+}=\bigcup_{n=1}^{\infty} D_{n}
$$

and

$$
\left(A_{n}-A_{n}\right)_{+}=\bigcup_{k=1}^{n} D_{k},
$$

for all $n \in \mathbb{N}$. Also, although we allow for the possibility that $D_{1}=\emptyset$, we do assume that the sequence $\left(\left(A_{n}-A_{n}\right)_{+}\right)_{n=1}^{\infty}$ is strictly increasing, which is to say that

$$
D_{n} \neq \emptyset \text {, for all } n \geq 2 \text {. }
$$

Note that it follows from (4.4) that the union $\cup_{n=1}^{\infty} D_{n}$ is disjoint.

Now, let $\left(g_{n}\right)_{n=1}^{\infty}$ be a sequence in $\ell^{2}(\mathbb{N})$, with $0<\left|g_{n+1}\right| \leq\left|g_{n}\right|$, for all $n \geq 1$. We assume that

$$
\sum_{n=1}^{\infty}\left|D_{n}\right| \cdot\left|g_{n}\right|^{2}<\infty
$$

Using (4.5), we let $f \in L^{2}(\mathbb{T})$ be any function whose Fourier transform $\widehat{f}$ on $\{0\} \cup(A-A)_{+}$is given by

$$
\begin{aligned}
& \widehat{f}(0)=0, \text { and } \\
& \widehat{f}(k)=g_{n}, \text { when } n \geq 1 \text { and } k \in D_{n} .
\end{aligned}
$$

Note that if $D_{1}=\emptyset$, then owing to (4.7) we need only take $n \geq 2$ in definition (4.9). The definition makes sense because (4.8) and (4.9) give

$$
\sum_{n \in(A-A)_{+}}|\widehat{f}(n)|^{2}=\sum_{n=1}^{\infty}\left|D_{n}\right| \cdot\left|g_{n}\right|^{2}<\infty .
$$

We now choose $\left(g_{n}\right)_{n=1}^{\infty}$ in a particular way. Let $r, r^{\prime} \in \mathbb{N}$ with $r^{\prime} \leq r$. We will want, for all $b_{1}, b_{2}, \ldots, b_{r^{\prime}} \in \mathbb{R}$,

$$
\sum_{n=-\infty}^{\infty} \frac{|\widehat{f}(n)|^{2}}{\sum_{i=1}^{r^{\prime}} d_{\mathbb{Z}}\left(b_{i} n\right)^{r}}=\infty
$$

For this, it would suffice to have

$$
\sum_{n \in(A-A)_{+}} \frac{|\widehat{f}(n)|^{2}}{\sum_{i=1}^{r^{\prime}} d_{\mathbb{Z}}\left(b_{i} n\right)^{r}}=\infty
$$


for all $b_{1}, b_{2}, \ldots, b_{r^{\prime}} \in \mathbb{R}$.

Now, let $b_{1}, b_{2}, \ldots, b_{r^{\prime}} \in \mathbb{R}$ be given. Put, for all $n \in \mathbb{N}$,

$$
\mu_{n}=\frac{1}{\sum_{i=1}^{r^{\prime}} d_{\mathbb{Z}}\left(b_{i} n\right)^{r}} \in(0, \infty] .
$$

In order to consider the cases $D_{1}=\emptyset$ and $D_{1} \neq \emptyset$ simultaneously, we put $m_{0}=1$ if $D_{1} \neq \emptyset$, and $m_{0}=2$ if $D_{1}=\emptyset$. We have

$$
\sum_{n \in(A-A)_{+}} \frac{|\widehat{f}(n)|^{2}}{\sum_{i=1}^{r^{\prime}} d_{\mathbb{Z}}\left(b_{i} n\right)^{r}}=\sum_{k=1}^{\infty} \sum_{j \in D_{k}}|\widehat{f}(j)|^{2} \mu_{j}=\lim _{m \rightarrow \infty} \sum_{k=1}^{m} \sum_{j \in D_{k}}|\widehat{f}(j)|^{2} \mu_{j} .
$$

Now let $m \in \mathbb{N}$ with $m>1$ be given, for the moment. Then, by (4.6) we have

$$
\left(A_{m}-A_{m}\right)_{+}=\bigcup_{k=1}^{m} D_{k}=\bigcup_{k=m_{0}}^{m} D_{k}=\left\{y_{1}, y_{2}, \ldots, y_{u}\right\}
$$

where

$$
\begin{gathered}
y_{1}, \ldots, y_{u_{1}} \in D_{m_{0}}, y_{u_{1}+1}, \ldots, y_{u_{2}} \in D_{m_{0}+1}, \ldots, \text { and } \\
y_{u_{m-1}} \in D_{m-1}, y_{u_{m-1}+1}, \ldots, y_{u_{m}} \in D_{m} .
\end{gathered}
$$

Note that $u_{m}=u=\left|\left(A_{m}-A_{m}\right)_{+}\right|$. Observe from the definition of $f$ that as $\left|g_{1}\right| \geq\left|g_{2}\right| \geq \cdots \geq\left|g_{m}\right|$

$$
\begin{aligned}
\left|\widehat{f}\left(y_{1}\right)\right|=\cdots=\left|\widehat{f}\left(y_{u_{1}}\right)\right| & \geq\left|\widehat{f}\left(y_{u_{1}+1}\right)\right|=\cdots \geq \cdots \\
= & \left|\widehat{f}\left(y_{u_{m-1}}\right)\right| \geq\left|\widehat{f}\left(y_{u_{m-1}+1}\right)\right|=\cdots=\left|\widehat{f}\left(y_{u_{m}}\right)\right| .
\end{aligned}
$$


Now, using (4.6), and applying Lemma 4.1 with $p=1$ and $q=m$, we have

$$
\begin{aligned}
& \sum_{j \in\left(A_{m}-A_{m}\right)_{+}}|\widehat{f}(j)|^{2} \mu_{j} \\
& =\sum_{k=m_{0}}^{m} \sum_{j \in D_{k}}|\widehat{f}(j)|^{2} \mu_{j} \\
& =\sum_{k=1}^{u}\left|\widehat{f}\left(y_{k}\right)\right|^{2} \mu_{y_{k}} \\
& \geq \sum_{k=1}^{u-1}\left(\left|\widehat{f}\left(y_{k}\right)\right|^{2}-\left|\widehat{f}\left(y_{k+1}\right)\right|^{2}\right)\left(\mu_{y_{1}}+\mu_{y_{2}}+\cdots+\mu_{y_{k}}\right) \\
& =\sum_{\ell=1}^{m-1}\left(\left|\widehat{f}\left(y_{u_{\ell}}\right)\right|^{2}-\left|\widehat{f}\left(y_{u_{\ell}+1}\right)\right|^{2}\right)\left(\mu_{y_{1}}+\mu_{y_{2}}+\cdots+\mu_{y_{u_{\ell}}}\right) \\
& =\sum_{\ell=m_{0}}^{m-1}\left(\left|g_{\ell}\right|^{2}-\left|g_{\ell+1}\right|^{2}\right)\left(\mu_{y_{1}}+\mu_{y_{2}}+\cdots+\mu_{y_{u_{\ell}}}\right) \\
& =\sum_{\ell=m_{0}}^{m-1}\left(\left|g_{\ell}\right|^{2}-\left|g_{\ell+1}\right|^{2}\right) \sum_{j=1}^{u_{\ell}} \frac{1}{\sum_{i=1}^{r^{\prime}} d_{\mathbb{Z}}\left(b_{i} y_{j}\right)^{r}} \\
& =\sum_{\ell=m_{0}}^{m-1}\left(\left|g_{\ell}\right|^{2}-\left|g_{\ell+1}\right|^{2}\right) \sum_{j \in\left(A_{\ell}-A_{\ell}\right)_{+}} \frac{1}{\sum_{i=1}^{r^{\prime}} d_{\mathbb{Z}}\left(b_{i} j\right)^{r}} .
\end{aligned}
$$

It now follows from Lemma 4.4, (4.5), (4.12) and (4.14) that

$$
\begin{aligned}
& \sum_{n \in(A-A)_{+}} \frac{|\widehat{f}(n)|^{2}}{\sum_{i=1}^{r^{\prime}} d_{\mathbb{Z}}\left(b_{i} n\right)^{r}} \\
= & \lim _{m \rightarrow \infty} \sum_{j \in\left(A_{m}-A_{m}\right)_{+}}|\widehat{f}(j)|^{2} \mu_{j} \\
\geq & \sum_{\ell=m_{0}}^{\infty}\left(\left|g_{\ell}\right|^{2}-\left|g_{\ell+1}\right|^{2}\right) \sum_{j \in\left(A_{\ell}-A_{\ell}\right)_{+}} \frac{1}{\sum_{i=1}^{r^{\prime}} d_{\mathbb{Z}}\left(b_{i} j\right)^{r}} \\
\geq & \left\{\begin{array}{l}
J\left(r, r^{\prime}\right) \sum_{\ell=m_{0}}^{\infty}\left(\left|g_{\ell}\right|^{2}-\left|g_{\ell+1}\right|^{2}\right)\left|A_{\ell}\right|^{r / r^{\prime}}, \\
K(r) \sum_{\ell=m_{0}}^{\infty}\left(\left|g_{\ell}\right|^{2}-\left|g_{\ell+1}\right|^{2}\right)\left|A_{\ell}\right| \log \left|A_{\ell}\right|, \quad \text { if } r=r^{\prime} .
\end{array}\right.
\end{aligned}
$$

Note that (4.15) applies for all choices of $b_{1}, b_{2}, \ldots, b_{r^{\prime}} \in \mathbb{R}$ and that the right hand side of $(4.15)$ is independent of $b_{1}, b_{2}, \ldots, b_{r^{\prime}}$. Also, $m_{0}=1$ or $m_{0}=2$ according as to whether 
we have $D_{1} \neq \emptyset$ or $D_{1}=\emptyset$ respectively. We will apply (4.15) to give examples of functions that cannot be expressed as a sum of a prescribed number of differences of order $s$.

Theorem 4.1. Let $\left(A_{n}\right)_{n=1}^{\infty}$ be an increasing sequence of non-empty finite subsets of $\mathbb{Z}$ and put $A=\cup_{n=1}^{\infty} A_{n}$. We assume that the sequence $\left(\left(A_{n}-A_{n}\right)_{+}\right)_{n=1}^{\infty}$ of sets is strictly increasing. Let the sets $D_{n}$ be as in (4.4), and let $\left(g_{n}\right)_{n=1}^{\infty}$ be a sequence in $\ell^{2}(\mathbb{N})$ such that $0<\left|g_{n+1}\right| \leq\left|g_{n}\right|$ for all $n$. We assume that

$$
\sum_{n=1}^{\infty}\left|D_{n}\right| \cdot\left|g_{n}\right|^{2}<\infty .
$$

Let $f$ be any function in $L^{2}(\mathbb{T})$ whose Fourier transform on $\{0\} \cup(A-A)_{+}$is given by

$$
\widehat{f}(0)=0 \text { and } \widehat{f}(k)=g_{n},
$$

whenever $k \in D_{n}$. Put $m_{0}=1$ if $D_{1} \neq \emptyset$, and put $m_{0}=2$ if $D_{1}=\emptyset$. Then, the following hold.

(i) For all $s \in \mathbb{N}$, the function $f$ is a sum of $2 s+1$ differences of order $s$.

(ii) If $r^{\prime}, s \in \mathbb{N}$ and $r^{\prime}<2 s$, and if

$$
\sum_{\ell=m_{0}}^{\infty}\left(\left|g_{\ell}\right|^{2}-\left|g_{\ell+1}\right|^{2}\right)\left|A_{\ell}\right|^{2 s / r^{\prime}}=\infty,
$$

then $f$ is not a sum of $r^{\prime}$ differences of order $s$.

(iii) If $s \in \mathbb{N}$ and

$$
\sum_{\ell=m_{0}}^{\infty}\left(\left|g_{\ell}\right|^{2}-\left|g_{\ell+1}\right|^{2}\right)\left|A_{\ell}\right| \log \left|A_{\ell}\right|=\infty,
$$

$f$ is not a sum of $2 s$ differences of order $s$.

Proof. (i) As $f \in L^{2}(\mathbb{T})$ and $\widehat{f}(0)=0, f$ is sum of $2 s+1$ differences of order $s$ by Theorem 8 of $[9]$.

(ii) As noted in (4.5), $(A-A)_{+}=\cup_{n=1}^{\infty}\left(A_{n}-A_{n}\right)_{+}$. We take $r=2 s$ in (4.15), and assume $r^{\prime}<2 s$. Then we deduce from the above assumptions and (4.15) that for all $b_{1}, b_{2}, \ldots, b_{r^{\prime}} \in \mathbb{R}$,

$$
\sum_{n \in(A-A)_{+}} \frac{|\widehat{f}(n)|^{2}}{\sum_{i=1}^{r^{\prime}} d_{\mathbb{Z}}\left(b_{i} n\right)^{2 s}}=\infty .
$$

It now follows from (iii) in Theorem 2.1 that $f$ is not a sum of $r^{\prime}$ differences of order $s$.

(iii) We proceed along the lines of (ii), but we take $r=r^{\prime}=2 s$ and use the second inequality in (4.15). We deduce that for all $b_{1}, b_{2}, \ldots, b_{2 s} \in \mathbb{R}$,

$$
\sum_{n \in(A-A)_{+}} \frac{|\widehat{f}(n)|^{2}}{\sum_{i=1}^{2 s} d_{\mathbb{Z}}\left(b_{i} n\right)^{2 s}}=\infty .
$$

Again using (iii) of Theorem 2.1, we deduce that $f$ is not a sum of $2 s$ difference of order $s$. 
In Theorem 4.1, the functions $f \in L^{2}(\mathbb{T})$ satisfy $\widehat{f}(0)=0$ and are specified in addition by the values of $\widehat{f}$ on sets of the form $(A-A)_{+}$. The question arises as to how much freedom there is in constructing these sets.

In the following result, we apply Theorem 4.1 where the sets $A_{n}$ and $\left(A_{n}-A_{n}\right)_{+}$each form an arithmetic progression, the sets $D_{n}$ have polynomial growth, and $(A-A)_{+}$forms an arithmetic progression.

Theorem 4.2. Let $d, p, r^{\prime}, s \in \mathbb{N}$ with $r^{\prime}<2 s$, and let $\alpha \in \mathbb{R}$ with $p / 2<\alpha \leq p s / r^{\prime}$. Then, there is a function $f \in L^{2}(\mathbb{T})$ whose Fourier transform $\widehat{f}$ on $\{0\} \cup\{d, 2 d, 3 d, \ldots\}$ is given by

$$
\begin{aligned}
& \widehat{f}(0)=0, \text { and } \\
& \widehat{f}(k)=\frac{1}{n^{\alpha}},
\end{aligned}
$$

if $k \in\left\{(n-1)^{p} d,(n-1)^{p} d+1,\left(n^{p}-1\right) d-1\right\}$ and $n \in \mathbb{N}$ with $n \geq 2$.

Any such function $f$ is a sum of $2 s+1$ differences of order $s$, but $f$ is not a sum of $r^{\prime}$ differences of order $s$. In particular, if $p / 2<\alpha \leq p s /(2 s-1), f$ is not a sum of $2 s-1$ differences of order $s$.

Proof. Assuming for the moment that $f \in L^{2}(\mathbb{T})$, as $\widehat{f}(0)=0, f$ is a sum of $2 s+1$ differences of order $s$, by Theorem 8 in [9].

Now, let $A_{n}=\left\{d, 2 d, 3 d, \ldots, n^{p} d\right\}$ for $n \in \mathbb{N}$. Then, $D_{1}=\left(A_{1}-A_{1}\right)_{+}=\emptyset,\left(A_{n}-A_{n}\right)_{+}=$ $\left\{d, 2 d, 3 d, \ldots,\left(n^{p}-1\right) d\right\}$ for $n \geq 2$. We see that

$$
\left(A_{n}-A_{n}\right)_{+} \cap\left(A_{n-1}-A_{n-1}\right)_{+}^{c}=D_{n}=\left\{(n-1)^{p} d,\left((n-1)^{p}+1\right) d, \ldots,\left(n^{p}-1\right) d\right\},
$$

for $n \geq 2$. We have

$$
\left|D_{n}\right|=n^{p}-(n-1)^{p} \leq p n^{p-1} .
$$

Let $p / 2<\alpha \leq p s / r^{\prime}$ and put $g_{n}=1 / n^{\alpha}$ for all $n \in \mathbb{N}$. Then $2 \alpha-p>0$ and so

$$
\sum_{n=1}^{\infty}\left|D_{n}\right| \cdot\left|g_{n}\right|^{2} \leq p \sum_{n=1}^{\infty} \frac{1}{n^{2 \alpha-p+1}}<\infty .
$$

Also, $1 / \ell^{2 \alpha}-1 /(\ell+1)^{2 \alpha} \geq 2 \alpha /(\ell+1)^{2 \alpha+1}$ and $1 /(\ell+1) \geq 1 / 2 \ell$ for all $\ell \in \mathbb{N}$. Hence, putting $g_{\ell}=1 / n^{\alpha}$, we see that there is $c>0$ such that

$$
\begin{aligned}
\sum_{\ell=1}^{\infty}\left(\left|g_{\ell}\right|^{2}-\left|g_{\ell+1}\right|^{2}\right)\left|A_{\ell}\right|^{2 s / r^{\prime}} & \geq 2 \alpha \sum_{\ell=1}^{\infty} \frac{\ell^{2 p s / r^{\prime}}}{(\ell+1)^{2 \alpha+1}} \\
& \geq c \sum_{\ell=1}^{\infty} \frac{1}{\ell^{2 \alpha+1-2 p s / r^{\prime}}} \\
& =\infty
\end{aligned}
$$

as $2 \alpha+1-2 p s / r^{\prime} \leq 1$. We deduce from (4.16) that there are functions $f \in L^{2}(\mathbb{T})$ satisfying (4.16), and it then follows by (4.17) and by (i) of Theorem 4.1 that any such function $f$ is not a sum of $r^{\prime}$ differences of order $s$. The particular case is when $r^{\prime}=2 s-1$. 
Note that in Theorem 4.2, it may happen that $r^{\prime}<s$. In this case, there is $\alpha$ such that $p<\alpha \leq p s / r^{\prime}$. Theorem 4.2 still applies but in this case we have $\sum_{n=1}^{\infty}\left|D_{n}\right| \cdot\left|g_{n}\right| \leq$ $p \sum_{n=1}^{\infty} 1 / n^{\alpha-p+1}<\infty$. So, we see that there are functions $f$ as in the conclusion of Theorem 4.2 that have an absolutely convergent Fourier series. Using this observation and Theorem 4.2 we see that when $r^{\prime}<s$, there are continuous functions in $L^{2}(\mathbb{T})$, each of which is a sum of $2 s+1$ differences of order $s$ but none of which is a sum of $r^{\prime}$ differences of order $s$.

It is not clear whether the function $f$ in Theorem 4.2 can be the sum of $2 s$ differences of order $s$. The following result gives examples of functions $f$ in $L^{2}(\mathbb{T})$ such that, for all $s \in \mathbb{N}$, $f$ is a sum of $2 s+1$ differences of order $s$ but $f$ is not a sum of $2 s$ differences of order $s$.

Theorem 4.3. Let $\left(A_{n}\right)_{n=1}^{\infty}$ be a strictly increasing sequence of non-empty finite subsets of $\mathbb{Z}$ and put $A=\cup_{n=1}^{\infty} A_{n}$. We assume that the sequence of sets $\left(\left(A_{n}-A_{n}\right)_{+}\right)_{n=1}^{\infty}$ also is strictly increasing. Let the sets $D_{n}$ be as in (4.4), Assume that there is $K>0$ and $\gamma \in[0,1)$ such that

$$
\left|D_{n}\right| \leq K(\log (n+1))^{\gamma}, \text { for all } n \in \mathbb{N} .
$$

Let $\beta \in \mathbb{R}$ with $(1+\gamma) / 2<\beta \leq 1$. Then, there is a function $f \in L^{2}(\mathbb{T})$ whose Fourier transform $\widehat{f}$ on $\{0\} \cup(A-A)_{+}$is given by

$$
\begin{aligned}
& \widehat{f}(0)=0, \text { and } \\
& \widehat{f}(k)=\frac{1}{\sqrt{n}(\log (n+1))^{\beta}},
\end{aligned}
$$

for $k \in D_{n}$ and $n \in \mathbb{N}$. Then, for any $s \in \mathbb{N}$, any such function $f$ is a sum of $2 s+1$ differences of order $s$, but $f$ is not a sum of $2 s$ differences of order $s$.

Proof. As noted in (4.5), $(A-A)_{+}=\cup_{n=1}^{\infty} D_{n}$ and this union is disjoint. Hence, we have

$$
\begin{aligned}
\sum_{n \in(A-A)_{+}}|\widehat{f}(n)|^{2} & =\sum_{n=1}^{\infty} \sum_{k \in D_{n}}|\widehat{f}(k)|^{2} \\
& \leq K \sum_{n=1}^{\infty} \frac{(\log (n+1))^{\gamma}}{n(\log (n+1))^{2 \beta}}, \text { by }(4.18) \\
& =K \sum_{n=1}^{\infty} \frac{1}{n(\log (n+1))^{2 \beta-\gamma}} \\
& <\infty
\end{aligned}
$$

as $2 \beta-\gamma>1$. It follows that there is $f \in L^{2}(\mathbb{T})$ satisfying (4.19). As $\widehat{f}(0)=0$, we deduce that $f$ is a sum of $2 s+1$ differences of order $s$ by [9, Theorem 8].

Put $g_{n}=1 / \sqrt{n}(\log (n+1))^{\beta}$ for all $n \in \mathbb{N}$, and observe that

$$
\begin{aligned}
& \left|g_{n}\right|^{2}-\left|g_{n+1}\right|^{2} \\
& =\frac{1}{n(\log (n+1))^{2 \beta}}-\frac{1}{(n+1)(\log (n+2))^{2 \beta}} \\
& \geq \frac{1}{n(n+1)} \cdot \frac{1}{(\log (n+2))^{2 \beta}} .
\end{aligned}
$$


Let $b_{1}, b_{2}, \ldots, b_{2 s} \in \mathbb{R}$. Note that as $\left(A_{n}\right)_{n=1}^{\infty}$ is strictly increasing and $A_{1} \neq \emptyset,\left|A_{n}\right| \geq n$ for all $n$. By the second inequality in (4.15) with $r=r^{\prime}=2 s$, and by (4.20), we see that for some $C(s)>0$,

$$
\begin{aligned}
\sum_{n \in(A-A)_{+}} \frac{|\widehat{f}(n)|^{2}}{\sum_{i=1}^{2 s} d_{\mathbb{Z}}\left(b_{i} n\right)^{2 s}} & \geq C(s) \sum_{n=m_{0}}^{\infty} \frac{n \log n}{n^{2}(\log n)^{2 \beta}} \\
& =C(s) \sum_{n=m_{0}}^{\infty} \frac{1}{n(\log n)^{2 \beta-1}} \\
& =\infty,
\end{aligned}
$$

as $\beta \leq 1$. Thus, it follows from Theorem 2.1 that the function $f$ is not a sum of $2 s$ differences of order $s$.

In the following result, the sequences of sets $\left(A_{n}\right)_{n=1}^{\infty},\left(\left(A_{n}-A_{n}\right)_{+}\right)_{n=1}^{\infty}$ and $\left(D_{n}\right)_{n=1}^{\infty}$ increase in size geometrically, and $(A-A)_{+}=\mathbb{N}$.

Theorem 4.4. Let $r^{\prime}, s, b \in \mathbb{N}$ with $r^{\prime}<2 s$ and $b \geq 2$. Also, let $0<\delta \leq b^{2 s / r^{\prime}}-b$. Then, there is a function in $L^{2}(\mathbb{T})$ such that $\widehat{f}(0)=0$ and for all $n \in \mathbb{N}$ with $n \geq 2$, we have

$$
\widehat{f}(k)=\frac{1}{(b+\delta)^{n / 2}}, \text { for all } k \in\left\{b^{n-2}, b^{n-2}+1, \ldots, b^{n-1}-1\right\} .
$$

Then, if $f$ is any such function in $L^{2}(\mathbb{T}), f$ is not a sum of $r^{\prime}$ differences of order $s$.

Proof. Let $A_{n}=\left\{1,2,3, \ldots, b^{n-1}\right\}$, for $n \in \mathbb{N}$. We have $\left|A_{n}\right|=b^{n-1},\left(A_{1}-A_{1}\right)_{+}=\emptyset$, and

$$
\left(A_{n}-A_{n}\right)_{+}=\left\{1,2,3, \ldots, b^{n-1}-1\right\} \text { and }\left|\left(A_{n}-A_{n}\right)_{+}\right|=b^{n-1}-1 \text {, for } n \geq 2 .
$$

Putting $D_{n}=\left(A_{n}-A_{n}\right)_{+} \cap\left(A_{n-1}-A_{n-1}\right)_{+}^{c}$ for $n \geq 2$ we have in light of (4.4) that

$$
D_{1}=\emptyset, D_{n}=\left\{b^{n-2}, b^{n-2}+1, \ldots, b^{n-1}-1\right\} \text { and }\left|D_{n}\right|=(b-1) b^{n-2} \text { for } n \geq 2 \text {. }
$$

Now as we are assuming (4.21), we see that $\widehat{f}(k)=1 /(b+\delta)^{n / 2}$ for all $k \in D_{n}$. Hence,

$$
\sum_{n=1}^{\infty}|\widehat{f}(n)|^{2}=\sum_{n \in(A-A)_{+}}|\widehat{f}(n)|^{2}=\sum_{n=2}^{\infty} \sum_{k \in D_{n}}|\widehat{f}(k)|^{2}=\frac{b-1}{b^{2}} \sum_{n=2}^{\infty}\left(\frac{b}{b+\delta}\right)^{n}<\infty .
$$

Thus, there is $f \in L^{2}(\mathbb{T})$ satisfying (4.21).

Now, put $g_{n}=1 /(b+\delta)^{n / 2}$, for all $n \in \mathbb{N}$. We have

$$
\begin{aligned}
\sum_{n=2}^{\infty}\left(\left|g_{n}\right|^{2}-\left|g_{n+1}\right|^{2}\right)\left|A_{n}\right|^{2 s / r^{\prime}} & =\sum_{n=2}^{\infty}\left(\frac{1}{(b+\delta)^{n}}-\frac{1}{(b+\delta)^{n+1}}\right)\left(b^{n-1}\right)^{2 s / r^{\prime}} \\
& =\frac{1}{b^{2 s / r^{\prime}}} \sum_{n=2}^{\infty}\left(\frac{1}{(b+\delta)^{n}}-\frac{1}{(b+\delta)^{n+1}}\right) b^{2 n s / r^{\prime}} \\
& =\frac{1}{b^{2 s / r^{\prime}}} \sum_{n=2}^{\infty}\left(\frac{1}{(b+\delta)^{n}}\left(1-\frac{1}{b+\delta}\right)\right) b^{2 n s / r^{\prime}} \\
& =\frac{1}{b^{2 s / r^{\prime}}}\left(\frac{b+\delta-1}{b+\delta}\right) \sum_{n=2}^{\infty}\left(\frac{b^{2 s / r^{\prime}}}{b+\delta}\right)^{n}
\end{aligned}
$$


As we are assuming $0<\delta \leq b^{2 s / r^{\prime}}-b$, we have $b^{2 s / r^{\prime}} /(b+\delta) \geq 1$, and we deduce from (4.22) that $\sum_{n=2}^{\infty}\left(\left|g_{n}\right|^{2}-\left|g_{n+1}\right|^{2}\right)\left|A_{n}\right|^{2 s / r^{\prime}}=\infty$. Then, (4.15) gives

$$
\sum_{n=1}^{\infty} \frac{|\widehat{f}(n)|^{2}}{\sum_{i=1}^{r^{\prime}} d_{\mathbb{Z}}\left(b_{i} n\right)^{2 s}}=\infty
$$

and Theorem 2.1 implies that $f$ is not a sum of $r^{\prime}$ differences of order $s$.

In the following result, in contrast to Theorems 4.2 and 4.4 , the function $\widehat{f}$ is specified only on arbitrarily "sparse" subset of $\mathbb{N}$, but there is the more stringent restriction that $r^{\prime}<s$, instead of $r^{\prime}<2 s$.

Theorem 4.5. Let $r^{\prime}, s \in \mathbb{N}$ with $r^{\prime}<s$ and let $\theta \in \mathbb{R}$ with $1<\theta \leq s / r^{\prime}$. Let $\left(q_{n}\right)_{n=0}^{\infty}$ be a strictly increasing sequence in $\mathbb{N}$ such that $q_{0}=1$ and $q_{n}>2 q_{n-1}-1$, for all $n \in \mathbb{N}$. Then, there is a function $f$ in $L^{2}(\mathbb{T})$ such that

$$
\widehat{f}(0)=0 \text { and } \widehat{f}(k)=\frac{1}{n^{\theta}},
$$

whenever $n \in \mathbb{N}$ and $k \in\left\{q_{n}-q_{m}: 0 \leq m<n\right\}$. Then, if $f$ is any such function in $L^{2}(\mathbb{T})$, $f$ is not a sum of $r^{\prime}$ differences of order $s$.

Proof. Let $A_{n}=\left\{1, q_{1}, q_{2}, \ldots, q_{n}\right\}$, and let the sets $D_{n}$ be as given in (4.4), for all $n \in \mathbb{N}$. Note that $\left|A_{n}\right|=n+1$ and that $D_{n} \neq \emptyset$ for all $n \in \mathbb{N}$. Now, the assumption on the sequence $\left(q_{n}\right)_{n=0}^{\infty}$ implies that, for a given $n \in \mathbb{N}$ with $n \geq 2$, each of the distinct numbers $q_{n}-q_{m}$ with $0 \leq m<n$ is greater than any number of the form $q_{\ell}-q_{k}$ with $0 \leq k<\ell \leq n-1$. Consequently, we see that

$$
D_{n}=\left\{q_{n}-q_{m}: 0 \leq m<n\right\} \text { and }\left|D_{n}\right|=n .
$$

We put $A=\bigcup_{n=1}^{\infty} A_{n}$ and then, comparing with (4.5), we have

$$
(A-A)_{+}=\bigcup_{n=1}^{\infty} D_{n}=\left\{q_{n}-q_{m}: m, n \in\{0\} \cup \mathbb{N} \text { with } m<n\right\} .
$$

Now, let's put $\widehat{f}(0)=0$ and if $k \in D_{n}$ for some (necessarily unique) $n \in \mathbb{N}$, put $\widehat{f}(k)=1 / n^{\theta}$ for all $k \in D_{n}$. This defines $\widehat{f}$ on $(A-A)_{+}$and we have

$$
\sum_{n \in(A-A)_{+}}|\widehat{f}(n)|^{2}=\sum_{n=1}^{\infty} \sum_{k \in D_{n}}|\widehat{f}(k)|^{2}=\sum_{n=1}^{\infty} \frac{n}{n^{2 \theta}} \leq \sum_{n=1}^{\infty} \frac{1}{n^{2 \theta-1}}<\infty,
$$

as $\theta>1$. Hence, there is $f \in L^{2}(\mathbb{T})$ satisfying the above conditions. 
Now, put $g_{n}=1 / n^{\theta}$ for all $n \in \mathbb{N}$. We have

$$
\begin{aligned}
& \sum_{\ell=1}^{\infty}\left(\left|g_{\ell}\right|^{2}-\left|g_{\ell+1}\right|^{2}\right)\left|A_{\ell}\right|^{2 s / r^{\prime}} \\
& =\sum_{\ell=1}^{\infty}\left(\frac{1}{\ell^{2 \theta}}-\frac{1}{(\ell+1)^{2 \theta}}\right)(\ell+1)^{2 s / r^{\prime}} \\
& \geq 2 \theta \sum_{\ell=1}^{\infty} \frac{1}{(\ell+1)^{2 \theta+1-2 s / r^{\prime}}} \\
& =\infty
\end{aligned}
$$

because $\theta \leq s / r^{\prime}$ implies that $2 \theta+1-2 s / r^{\prime} \leq 1$. We deduce from (4.21) that $f$ cannot be a sum of $r^{\prime}$ differences of order $s$.

\section{Conclusion}

In constructing functions $f$ such as those in Theorem 4.1, there are two notable restrictions. On the one hand, for having $f \in L^{2}(\mathbb{T})$, we need the sequence $\left(g_{n}\right)_{n=1}^{\infty}$ asymptotically to have "small" terms so as to satisfy condition (4.8), which ensures the convergence of a series. This condition is determined by the rate of increase of the sets in the sequence $\left(\left(A_{n}-A_{n}\right)_{+}\right)_{n=1}^{\infty}$. On the other hand, we need the sequence $\left(\left|g_{n}\right|^{2}-\left|g_{n+1}\right|^{2}\right)_{n=1}^{\infty}$ asymptotically to have relatively "large" terms, to allow the divergence of series such as are in (4.15) and (4.17). The rate of increase of the sets in $\left(\left(A_{n}-A_{n}\right)_{+}\right)_{n=1}^{\infty}$ is lowest when the points of $A$ are in arithmetic progression, and is the highest when they are in a geometric progression. So, we see that with the methods here there can be fewer restrictions in constructing the functions $f$ when their Fourier transforms are supported by "regularly spaced" sets of the form $(A-A)_{+}$, rather than by "irregularly spaced" sets. Thus, in Theorem 4.5, there is a restriction on $r^{\prime}$ and $s$ that $r^{\prime}<s$, while in Theorem 4.4 we have $(A-A)_{+}=\mathbb{N}$ and the corresponding restriction on $r^{\prime}$ and $s$ is that $r^{\prime}<2 s$. Note that in Theorem 4.5, it can be shown that the set $(A-A)_{+}$is "sparse" in $\mathbb{N}$ in that it satisfies the equation

$$
\lim _{n \rightarrow \infty} \frac{1}{n} \mid\left\{j: 1 \leq j \leq n \text { and } j \in(A-A)_{+}\right\} \mid=0,
$$

and is thus a set of density zero in $\mathbb{N}$.

\section{References}

[1] Y. Bugeaud, Sets of exact approximation order by rational numbers, Math. Annalen, 327, (2003),171-190.

[2] Y. Bugeaud, Sets of exact approximation order by rational numbers III, Acta Arith. 146, (2011), 177-193.

[3] Y. Bugeaud, Distribution Modulo One and Diophantine Approximation, Cambridge Tracts in Mathematics 193, Cambridge University Press, Cambridge, 2012. 
[4] R. W. S. Cassels, Simultaneous Diophantine Approximation II, Proc. Lond. Math. Soc., $5,(1955), 537-577$.

[5] R. W. S. Cassels, An Introduction to Diophantine Approximation, Cambridge Tracts in Mathematics and Mathematical Pghyscis No.45, Cambridge University Press, Cambridge, 1957.

[6] I. M. Johnstone and M. Raimondo, Periodic boxcar deconvolution and Diophantine approximation, Annals of Statistics, 32 (2004), 1781-1804.

[7] R. E. Edwards, Fourier series, vol. 1, Holt, Rinehart and Winston.

[8] G. Meisters and W. Schmidt, Translation invariant linear forms on $L^{2}(G)$ for compact abelian groups G, Journ. Func. Anal. 12 (1973),199-210.

[9] R. Nillsen, Banach spaces of functions and distributions characterized by singular integrals involving the Fourier transform, Journ. Func. Anal. 110 (1992), 73-95.

[10] R. Nillsen, Difference spaces and Invariant Linear Forms, Lecture Notes in Mathematics 1586, Springer-Verlag, New York, 1994.

[11] R. Nillsen, Differentiate and make waves, Expositiones Math. 14, 1996, 57-84.

[12] R. Nillsen and S. Okada, Sharp results concerning the expression of functions as sums of finite differences, Proc. Lond. Math. Soc., (3) 88 (2004), 479-504.

[13] I. Niven, Irrational Numbers, Carus Mathematical Monographs 11, Mathematical Association of America, Washington DC, 1967.

[14] J. C. Oxtoby, Measure and Category, second edition, Springer-Verlag, Berlin, 1980.

[15] M. Pensky and T. Sapatinas, Multichannel boxcar deconvolution with a growing number of channels, Electronic Journ. of Statistics, 5 (2011), 53-52.

[16] W. M. Schmidt, Diophantine Approximation, Lecture Notes in Mathematics, 785, Springer-Varlag, Berlin, 1980.

[17] T. Tao and V. Vu, Additive Combinatorics, Cambridge University Press, Cambridge, 2006. 